\title{
FP-ADMET: a compendium of fingerprint-based ADMET prediction models
}

\author{
Vishwesh Venkatraman * (D)
}

\begin{abstract}
Motivation: The absorption, distribution, metabolism, excretion, and toxicity (ADMET) of drugs plays a key role in determining which among the potential candidates are to be prioritized. In silico approaches based on machine learning methods are becoming increasing popular, but are nonetheless limited by the availability of data. With a view to making both data and models available to the scientific community, we have developed FPADMET which is a repository of molecular fingerprint-based predictive models for ADMET properties.

Summary: In this article, we have examined the efficacy of fingerprint-based machine learning models for a large number of ADMET-related properties. The predictive ability of a set of 20 different binary fingerprints (based on substructure keys, atom pairs, local path environments, as well as custom fingerprints such as all-shortest paths) for over 50 ADMET and ADMET-related endpoints have been evaluated as part of the study. We find that for a majority of the properties, fingerprint-based random forest models yield comparable or better performance compared with traditional 2D/3D molecular descriptors.
\end{abstract}

Availability: The models are made available as part of open access software that can be downloaded from https:// gitlab.com/vishsoft/fpadmet.

Keywords: ADMET, Machine learning, Molecular fingerprints

\section{Introduction}

Properties such as absorption, distribution, metabolism, excretion and toxicity (ADMET), are an important component of pharmaceutical drug design. It is often reported that the failure to meet requisite ADMET criteria are a common cause for the high attrition rates of drug candidates [1]. Early ADMET profiling is indeed desirable so as to mitigate the risk of attrition. Various medium and high-throughput in vitro ADMET screens have therefore been developed, that have contributed to the available experimental data. These are nonetheless quite expensive especially when thousands of compounds are involved. Furthermore, reducing animal testing has now become a priority.

*Correspondence: vishwesh.venkatraman@ntnu.no Norwegian University of Science and Technology, Realfagbygget, Gløshaugen, Høgskoleringen, 7491 Trondheim, Norway
With the aim of facilitating rapid and inexpensive means of ADMET profiling, various in silico tools have been developed [2]. Using databases of experimentally measured ADMET properties [3], various quantitative structure-activity/property relationship (QSAR/QSPR) models have been generated that can predict a range of ADMET properties for novel chemical entities. Other efforts have made use of ADMET predictions to evaluate drug-likeness of a compound $[4,5]$. While some of the models are available as part of commercial software packages based on proprietary datasets, there has been a significant push for open source software and web services [6-12].

Among the popular services, ADMETLab [12] offers 53 prediction models that are calculated using a multi-task graph attention network and operates on graph-structured data. The method is able to generate customized fingerprints from the general features original author(s) and the source, provide a link to the Creative Commons licence, and indicate if changes were made. The images or other third party material in this article are included in the article's Creative Commons licence, unless indicated otherwise in a credit line to the material. If material is not included in the article's Creative Commons licence and your intended use is not permitted by statutory regulation or exceeds the permitted use, you will need to obtain permission directly from the copyright holder. To view a copy of this licence, visit http://creativecommons.org/licenses/by/4.0/. The Creative Commons Public Domain Dedication waiver (http://creativeco mmons.org/publicdomain/zero/1.0/) applies to the data made available in this article, unless otherwise stated in a credit line to the data. 
for a specific task. Another web tool, SwissADME [9] evaluates pharmacokinetics, drug-likeness of small molecules. The predictions are based on a combination of fragmental methods (for solubility), as well as machine-learning based binary classification methods for other ADMET properties (cytochrome-P450 inhibitor, P-glycoprotein substrate). In ADMETSar [11], models for applications in both drug discovery and environmental risk assessment are built using MACCS and Morgan fingerprints. The toxicity models used in ProTox [13] are developed based on chemical similarities between compounds with known toxic effects and the presence of toxic fragments. Other models for hepatotoxicity, cytotoxicity, mutagenicity, and carcinogenicity rely on fingerprints (MACCS/ Morgan). Extended connectivity fingerprints form the basis for the prediction of 15 ADMET properties in the vNN server [10] where models are trained using variable nearest neighbourhood method. pkCSM [6], on the other hand, uses graph-based signatures to develop predictive models of central ADMET properties. Other software such as MDCKPred [14], CarcinoPred-EL [15], CapsCarcino [16] focus on a single property such as the prediction of permeability coefficient and carcinogenic compounds. Overall, the molecular representations underlying these models include various molecular and physicochemical descriptors such as fingerprints, graph signatures, and other $2 \mathrm{D} / 3 \mathrm{D}$ indices $[17,18]$. Among these, fingerprint representations which are seen as an alternative to descriptors for QSPR studies, have been quite popular given their ease of computation and predictive value.

A number of fingerprints ranging from substructure/path to feature-class/circular have been proposed many of which are used in similarity searching $[19,20]$. For ADMET studies however, the fingerprints studied so far have largely been restricted to a select few. In this study, we have evaluated the predictive efficacy of 20 different fingerprints ranging from substructure and extended/functional connectivity fingerprints to various path based encodings (depth-first search, shortest path, local path environments) [21]. The fingerprint-based regression/classification models were calculated for over 50 ADMET and ADMETrelated endpoints (using data collated from various literature sources) and is to our knowledge one of the most comprehensive compilations analysed. For a majority of the endpoints, the prediction results were found to be comparable with more sophisticated descriptor formulations. Although the pharmacophore fingerprints yielded consistently poor results, others such as the PUBCHEM, MACCS and ECFP/ FCFP encodings were found to yield the best results for most properties. The models and related software have been bundled into a downloadable package and is released under the GNU license.

\section{Approach \\ Molecular representation}

In this study, we have examined 20 different fingerprints (see Table 1) that are routinely used as similarity search tools in drug discovery. The ECFP- and FCFP-class fingerprints are circular topological fingerprints, where the former focuses on the atom properties (e.g. atomic number, charge, hydrogen count), whereas in the functional connectivity FPs, the emphasis is on properties that relate to ligand binding (e.g. hydrogen donor/acceptor, polarity, aromaticity). MACCS and PUBCHEM fingerprints are substructure fingerprints that cover a wide range of features such as element counts and ring systems, atom pairing, or atom environment etc. Other fingerprints include path based fingerprints such as the depth-first search fingerprints (DFS), all-shortest path encoding (ASP), radial fingerprints (Molprint2D), topological atom pairs (AP2D) and triplets (AT2D), pharmacophore pair and triplet encodings as well as local path environments [21]. Fingerprint calculations were performed using in-house code written in Java and makes use of the Chemistry Development Kit library [22]. The software merges existing fingerprints in the library with those calculated by the software jCompoundMapper [21].

Table 1 Fingerprints used in this study to model different ADMET related properties

\begin{tabular}{lr}
\hline Fingerprint & Size \\
\hline MACCS & 166 \\
PUBCHEM & 881 \\
Klekota-Roth (KR) & 4860 \\
MOLPRINT (RAD2D) & 4096 \\
Atom pair (AP), atom triplet (AT) & 4096 \\
Local path environments (LSTAR) & 4096 \\
All-shortest path (ASP) & 4096 \\
Depth first search (DFS) & 4096 \\
Extended conectivity (ECFP: 0, 2, 4, 6) & 1024 \\
Functional class (FCFP: 0, 2, 4, 6) & 1024 \\
Pharmacophore: 2PPHAR/3PPHAR (2/3 point) & 4096 \\
ESTATE & 79 \\
\hline
\end{tabular}

Descriptions and implementation details of the different fingerprints are provided in the article by Hinselmann et al [21] and the references therein 
Table 2 Summary of the ADMET endpoints studied

\begin{tabular}{|c|c|c|c|c|}
\hline Endpoint & Model & \#Compounds & Group & Data source \\
\hline Blood brain barrier & $\mathrm{BC}$ & 7236 & Distribution & {$[3,31]$} \\
\hline Oral bioavailability & $B C$ & 1822 & Absorption & {$[3,32]$} \\
\hline Anticommensal effect & $B C$ & 1181 & Toxicity & {$[33,34]$} \\
\hline CYP450 (1A2) inhibition & $B C$ & 17119 & Metabolism & [35] \\
\hline CYP450 (2C19) inhibition & $B C$ & 17119 & Metabolism & [35] \\
\hline CYP450 (2C9) inhibition & $B C$ & 17119 & Metabolism & {$[35]$} \\
\hline CYP450 (2D6) inhibition & $B C$ & 17119 & Metabolism & [35] \\
\hline CYP450 (3A4) inhibition & $B C$ & 17119 & Metabolism & [35] \\
\hline CYP450 (2C8) inhibition & $\mathrm{BC}$ & 533 & Metabolism & [36] \\
\hline $\mathrm{HIA}$ & $B C$ & 1516 & Absorption & {$[3,37]$} \\
\hline BCRP inhibition & $\mathrm{BC}$ & 2799 & Metabolism & [38] \\
\hline Metabolic intrinsic clearance & $M C$ & 5278 & Excretion & [39] \\
\hline Human liver microsomal stability & $B C$ & 3654 & & {$[40]$} \\
\hline PGP inhibitor & $B C$ & 2930 & Distribution & {$[3,41]$} \\
\hline PGP substrate & $B C$ & 2198 & Distribution & {$[3,41]$} \\
\hline DMSO solubility & $B C$ & 59047 & & {$[42]$} \\
\hline Phosphate buffer solubility & $B C$ & 57584 & & [43] \\
\hline Skin sensitization (LLNA) & $B C$ & 1033 & Toxicity & [44] \\
\hline Skin sensitization (KeratinSens) & $B C$ & 190 & Toxicity & [44] \\
\hline Skin sensitization (HRIPT) & $B C$ & 138 & Toxicity & [44] \\
\hline Skin sensitization (h-CLAT) & $B C$ & 160 & Toxicity & [44] \\
\hline Skin sensitization (DPRA) & $B C$ & 194 & Toxicity & [44] \\
\hline Rat acute oral toxicity $\left(L_{50}\right)$ & $M C$ & 11363 & Toxicity & {$[3,45]$} \\
\hline AMES mutagenecity & $B C$ & 7950 & Toxicity & [46] \\
\hline Cytotoxicity (HepG2) & $B C$ & 6081 & Toxicity & [10] \\
\hline Cytotoxicity (CRL-7250 cell line) & $B C$ & 5241 & Toxicity & [47] \\
\hline Cytotoxicity (HACAT cell line) & $B C$ & 5241 & Toxicity & [47] \\
\hline Cytotoxicity (HEK cell line) & $B C$ & 5241 & Toxicity & [47] \\
\hline Cytotoxicity (NIK cell line) & $B C$ & 5241 & Toxicity & [47] \\
\hline DILI & $B C$ & 2478 & Toxicity & [48] \\
\hline Hemolytic toxicity (saponins) & $B C$ & 452 & Toxicity & [49] \\
\hline hERG cardiotoxicity & $B C$ & 7889 & Toxicity & [50] \\
\hline hERG liability & $B C$ & 9204 & & {$[51]$} \\
\hline Mitochondrial toxicity & $B C$ & 6467 & Toxicity & {$[52]$} \\
\hline Urinary tract toxicity & $B C$ & 213 & Toxicity & {$[53,54]$} \\
\hline Phototoxicity & $B C$ & 516 & Toxicity & {$[55]$} \\
\hline Phototoxicity & $B C$ & 1419 & Toxicity & [55] \\
\hline Toxic myopathy & $B C$ & 232 & Toxicity & {$[56]$} \\
\hline Myelotoxicity & $B C$ & 907 & Toxicity & [57] \\
\hline Phospholipidosis & $B C$ & 1719 & Toxicity & [58] \\
\hline Choleostasis & $B C$ & 1926 & Toxicity & [59] \\
\hline Rhabdomyolysis & $B C$ & 1504 & Toxicity & [60] \\
\hline Respiratory toxicity & $B C$ & 1241 & Toxicity & [61] \\
\hline Ototoxicity & $B C$ & 2612 & Toxicity & [62] \\
\hline MATE1 inhibition & $B C$ & 853 & Metabolism & [63] \\
\hline Hepatic steatosis & $B C$ & 512 & Toxicity & [64] \\
\hline Carcinogenecity & $B C$ & 1003 & Toxicity & [15] \\
\hline OATP1B1 inhibition & $B C$ & 1339 & Metabolism & [65] \\
\hline OATP2B1 inhibition & $B C$ & 230 & Metabolism & [65] \\
\hline
\end{tabular}


Table 2 (continued)

\begin{tabular}{|c|c|c|c|c|}
\hline Endpoint & Model & \#Compounds & Group & Data source \\
\hline OATP1B3 inhibition & $B C$ & 1249 & Metabolism & [65] \\
\hline BSEP inhibition & $\mathrm{BC}$ & 1634 & Metabolism & [66] \\
\hline OCT2 inhibition & $\mathrm{BC}$ & 907 & Metabolism & {$[67]$} \\
\hline PPB & $M C$ & 8103 & Distribution & {$[3,68]$} \\
\hline Elimination half-life Human & $M C$ & 2127 & Excretion & {$[69]$} \\
\hline Elimination half-life Mouse & $M C$ & 808 & Excretion & [69] \\
\hline Elimination half-life Rat & $M C$ & 1308 & Excretion & [69] \\
\hline
\end{tabular}

Here $B C$ and $M C$ refer to binary and multiclass classification respectively

OATP organic anion transporting polypeptide, CYP-450 cytochrome-P450, BCRP breast cancer resistance protein, BSEP bile salt export pump, DILI drug-induced liver injury, OCT organic cation transporter 2, MATE1 multidrug toxin extrusion transporter, $h E R G$ human Ether-á-go-go-related gene, $H I A$ human intestinal absorption, $P P B$ plasma protein binding, PGP p-glycoprotein, LLNA local lymph node assay, DPRA direct peptide reactivity assay, $h$-CLAT human cell line activation, $H R I P T$ human repeat insult patch test, HEK 293 human embryonic kidney 293 cell, MATE1 multidrug and toxin extrusion transporter 1

Table 3 Summary of the ADMET and other endpoints for which fingerprint-based regression models were evaluated

\begin{tabular}{|c|c|c|c|}
\hline Endpoint & \#Compounds & Group & Data source \\
\hline Aqueous solubility (logS) & 9982 & & [70] \\
\hline Intrinsic clearance $\left(\mathrm{CL}_{\text {int }}\right)$ & 244 & Excretion & [71] \\
\hline Skin penetration (log kp) & 211 & Toxicity & {$[72]$} \\
\hline Human serum albumin & 198 & & {$[73,74]$} \\
\hline Human placenta barrier (clearance index) & 88 & Distribution & [75] \\
\hline Cancer potency in mouse $\left(\mathrm{TD}_{50}\right)$ & 402 & Toxicity & [76] \\
\hline Cancer potency in rat $\left(\mathrm{TD}_{50}\right)$ & 511 & Toxicity & [76] \\
\hline Steady state volume distribution $\left(\mathrm{VD}_{s s}\right)$ & 1951 & Distribution & {$[3,77]$} \\
\hline Distribution coefficient (log D) & 7321 & & {$[3,78]$} \\
\hline Fraction unbound in human plasma & 2319 & Distribution & [79] \\
\hline Fraction unbound in the brain & 253 & Distribution & [80] \\
\hline Human liver microsomal clearance & 5348 & Excretion & [30] \\
\hline Rat liver microsomal clearance & 2166 & Excretion & [30] \\
\hline Mouse liver microsomal clearance & 790 & Excretion & [30] \\
\hline CACO-2 permeability & 2578 & Absorption & [30] \\
\hline $\mathrm{pK}_{a}$ & 11041 & & {$[81,82]$} \\
\hline MDCK cell line permeability & 701 & Absorption & [3] \\
\hline Human renal clearance $\left(C L_{r}\right)$ & 636 & Excretion & [83] \\
\hline Hemolytic toxicity $\left(\log H D_{50}\right)$ & 875 & Toxicity & [84] \\
\hline
\end{tabular}

MDCK Madin-Darby canine kidney

\section{Data curation}

Data for different endpoints were collected from previously published articles and databases with a primary source being the Online Chemical Database (OCHEM) [3]. The molecules were subsequently cleaned and duplicates (where present) were removed. Tables 2 and 3 lists the various endpoints and associated data sources considered in this study. Brief descriptions of the endpoints and the results from previous modelling efforts are provided in Additional file 1. Since, early identification of severe toxicity is a key requirement for the safety evaluation of drug candidates, we have evaluated a number of toxicity models covering a range of endpoints such as cardiac, hepatotoxicity, endocrine, urinary tract, carcinogenicity and cytotoxicity. While a majority of the models are binary classification models, for some endpoints such the metabolic intrinsic clearance, acute oral toxicity in rats, plasma protein binding and elimination half-life, multiclass models are proposed.

For other endpoints, regression models have been evaluated (see Table 3). These include the CACO-2 permeability which is commonly used to predict the 
Table 4 Performance metrics for the best performing fingerprint-based classification models

\begin{tabular}{|c|c|c|c|c|c|}
\hline \multirow[t]{2}{*}{ Endpoint } & \multirow[t]{2}{*}{$\mathrm{FP}$} & \multicolumn{2}{|c|}{ Calibration } & \multicolumn{2}{|c|}{ Validation } \\
\hline & & BACC & AUC & BACC & AUC \\
\hline Blood brain barrier & PUBCHEM & 0.82 & 0.90 & 0.81 & 0.92 \\
\hline Oral bioavailability & PUBCHEM & 0.71 & 0.77 & 0.71 & 0.78 \\
\hline Anticommensal effect & PUBCHEM & 0.76 & 0.82 & 0.74 & 0.81 \\
\hline CYP450 (1A2) & PUBCHEM & 0.85 & 0.93 & 0.85 & 0.93 \\
\hline CYP450 (2C19) & ECFP4 & 0.81 & 0.88 & 0.81 & 0.89 \\
\hline CYP450 (2C9) & PUBCHEM & 0.78 & 0.88 & 0.79 & 0.89 \\
\hline CYP450 (2D6) & FCFP4 & 0.73 & 0.86 & 0.73 & 0.87 \\
\hline CYP450 (3A4) & FCFP6 & 0.80 & 0.89 & 0.80 & 0.90 \\
\hline CYP450 (2C8) & PUBCHEM & 0.79 & 0.89 & 0.77 & 0.90 \\
\hline HIA & MACCS & 0.84 & 0.89 & 0.83 & 0.89 \\
\hline BCRP inhibition & FCFP4 & 0.89 & 0.95 & 0.90 & 0.96 \\
\hline Metabolic intrinsic clearance & FCFP4 & 0.74 & 0.82 & 0.74 & 0.84 \\
\hline $\begin{array}{l}\text { Human liver microsomal } \\
\text { stability }\end{array}$ & AT2D & 0.77 & 0.83 & 0.77 & 0.84 \\
\hline PGP inhibitor & PUBCHEM & 0.84 & 0.91 & 0.85 & 0.92 \\
\hline PGP substrate & ASP & 0.80 & 0.87 & 0.80 & 0.88 \\
\hline DMSO solubility & ECFP2 & 0.72 & 0.78 & 0.73 & 0.80 \\
\hline Phosphate buffer solubility & PUBCHEM & 0.79 & 0.87 & 0.79 & 0.87 \\
\hline Skin sensitization (LLNA) & PUBCHEM & 0.69 & 0.76 & 0.67 & 0.74 \\
\hline Skin sensitization (KeratinSens) & LSTAR & 0.64 & 0.65 & 0.57 & 0.60 \\
\hline Skin sensitization (HRIPT) & ECFPO & 0.70 & 0.74 & 0.67 & 0.72 \\
\hline Skin sensitization (hCLAT) & MACCS & 0.65 & 0.70 & 0.61 & 0.68 \\
\hline Skin sensitization (DPRA) & FCFP4 & 0.68 & 0.72 & 0.68 & 0.72 \\
\hline Rat acute oral toxicity $\left(\mathrm{LD}_{50}\right)$ & PUBCHEM & 0.69 & 0.78 & 0.68 & 0.81 \\
\hline AMES mutagenecity & PUBCHEM & 0.79 & 0.86 & 0.79 & 0.87 \\
\hline Cytotoxicity (HepG2) & AT2D & 0.78 & 0.85 & 0.78 & 0.85 \\
\hline Cytotoxicity (CRL-7250 cell line) & AT2D & 0.79 & 0.87 & 0.78 & 0.86 \\
\hline Cytotoxicity (HACAT cell line) & AT2D & 0.77 & 0.85 & 0.77 & 0.85 \\
\hline Cytotoxicity (HEK cell line) & PUBCHEM & 0.77 & 0.87 & 0.76 & 0.86 \\
\hline Cytotoxicity (NIK cell line) & PUBCHEM & 0.78 & 0.87 & 0.78 & 0.87 \\
\hline DILI & PUBCHEM & 0.78 & 0.86 & 0.79 & 0.88 \\
\hline Hemolytic toxicity (saponins) & FCFP6 & 0.84 & 0.88 & 0.85 & 0.90 \\
\hline hERG cardiotoxicity & FCFP6 & 0.79 & 0.86 & 0.80 & 0.88 \\
\hline hERG liability & PUBCHEM & 0.76 & 0.87 & 0.76 & 0.88 \\
\hline Mitochondrial toxicity & PUBCHEM & 0.79 & 0.90 & 0.77 & 0.90 \\
\hline Urinary tract toxicity & FCFP4 & 0.71 & 0.77 & 0.70 & 0.73 \\
\hline Phototoxicity in vitro & $\mathrm{KR}$ & 0.70 & 0.76 & 0.69 & 0.80 \\
\hline Phototoxicity human & PUBCHEM & 0.69 & 0.75 & 0.67 & 0.75 \\
\hline Toxic myopathy & DFS & 0.68 & 0.74 & 0.63 & 0.74 \\
\hline Myelotoxicity & FCFP4 & 0.72 & 0.79 & 0.71 & 0.80 \\
\hline phospholipidosis & FCFP2 & 0.78 & 0.86 & 0.77 & 0.88 \\
\hline Cholestasis & RAD2D & 0.67 & 0.73 & 0.67 & 0.74 \\
\hline Rhabdomyolysis & MACCS & 0.71 & 0.80 & 0.70 & 0.83 \\
\hline Respiratory toxicity & MACCS & 0.82 & 0.88 & 0.82 & 0.89 \\
\hline Ototoxicity & PUBCHEM & 0.69 & 0.74 & 0.67 & 0.72 \\
\hline MATE1 & DFS & 0.64 & 0.67 & 0.65 & 0.65 \\
\hline Hepatic steatosis & MACCS & 0.63 & 0.67 & 0.59 & 0.68 \\
\hline Carcinogenecity & PUBCHEM & 0.67 & 0.71 & 0.68 & 0.75 \\
\hline
\end{tabular}

Table 4 (continued)

\begin{tabular}{llllllll}
\hline Endpoint & FP & & \multicolumn{2}{c}{ Calibration } & & \multicolumn{2}{c}{ Validation } \\
& & & BACC & AUC & & BACC & AUC \\
\hline OATP1B1 inhibition & ECFP6 & 0.72 & 0.80 & & 0.73 & 0.82 \\
OATP2B1 inhibition & ECFP6 & 0.67 & 0.68 & & 0.65 & 0.70 \\
OATP1B3 inhibition & PUBCHEM & 0.74 & 0.83 & & 0.77 & 0.87 \\
BSEP inhibition & ECFP4 & 0.85 & 0.93 & & 0.88 & 0.95 \\
OCT2 inhibition & PUBCHEM & 0.73 & 0.81 & & 0.73 & 0.79 \\
PPB & PUBCHEM & 0.82 & 0.92 & & 0.84 & 0.92 \\
Elimination half-life Human & ASP & 0.75 & 0.86 & & 0.76 & 0.88 \\
Elimination half-life Mouse & ECFP2 & 0.74 & 0.86 & & 0.72 & 0.84 \\
Elimination half-life Rat & KR & 0.74 & 0.86 & & 0.74 & 0.83
\end{tabular}

The values reported are the balanced accuracies (BACC) and area under the ROC curve (AUC) (average of 3 independent runs) for the calibration/validation sets

absorption of orally administered drugs and other xenobiotics, the fraction of unbound drug in plasma, the liver microsomal clearance (typically used to predict hepatic clearance in humans), in vitro human skin permeability and the cancer potency. Models for other ADMET-related properties have also been studied. For instance, properties such as the dissociation constant $\left(\mathrm{pK}_{a}\right)$ affect solubility (log S), permeability, distribution coefficient $(\log \mathrm{D})$ and oral absorption. These in turn along with other properties such as the human serum albumin (HSA) binding impact pharmacokinetic behaviour and drug bioavailability.

\section{Modelling}

In order to build the models, the Random Forest algorithm [23] was chosen which is an ensemble learning method for both classification and regression. The algorithm makes use of bagging and feature randomness to build multiple decision trees (each trained on a random subset of data) and merges them together. The models were trained using the ranger [24] library in the statistical computing environment $R$ [25]. The number of trees used to compute the final average predicted value was set to 500 . For each endpoint, the data was split randomly into separate training (80\%) and test $(20 \%)$ sets. A fivefold cross-validation was used to identify the best performing model. In order to rule out any selection bias, we repeated random splitting 3 times and the results were averaged to gain an understanding of the variability. Furthermore, $y$-randomization tests were conducted to assess the robustness of the final model. To address the problem with unequal distribution of samples between classes, data augmentation of the minority class was carried out using the synthetic minority oversampling technique (SMOTE) [26]. 
Table 5 Performance metrics for the best performing fingerprint-based regression models

\begin{tabular}{|c|c|c|c|c|c|c|c|}
\hline \multirow[t]{2}{*}{ Endpoint } & \multirow[t]{2}{*}{ FP } & \multicolumn{3}{|c|}{ Calibration } & \multicolumn{3}{|c|}{ Validation } \\
\hline & & $\mathbf{R}^{2}$ & RMSE & MAE & $\mathrm{R}^{2}$ & RMSE & MAE \\
\hline $\log S$ & PUBCHEM & 0.77 & 1.15 & 0.81 & 0.78 & 1.12 & 0.78 \\
\hline Intrinsic clearance $\left(C L_{i n t}\right)$ & RAD2D & 0.48 & 0.83 & 0.65 & 0.29 & 1.02 & 0.82 \\
\hline Skin penetration $\left(\log k_{p}\right)$ & PUBCHEM & 0.73 & 0.60 & 0.48 & 0.75 & 0.56 & 0.43 \\
\hline Human serum albumin & AP2D & 0.71 & 0.33 & 0.23 & 0.69 & 0.39 & 0.26 \\
\hline Human placenta barrier & $\mathrm{KR}$ & 0.41 & 0.24 & 0.20 & 0.24 & 0.32 & 0.22 \\
\hline Cancer potency in mouse $\left(T D_{50}\right)$ & AT2D & 0.33 & 0.98 & 0.75 & 0.27 & 0.96 & 0.72 \\
\hline Cancer potency in rat $\left(T D_{50}\right)$ & AT2D & 0.41 & 1.08 & 0.83 & 0.35 & 1.14 & 0.87 \\
\hline Steady state volume distribution $\left(V D_{s s}\right)$ & ASP & 0.58 & 0.44 & 0.29 & 0.45 & 0.51 & 0.32 \\
\hline Distribution coefficient $(\log D)$ & PUBCHEM & 0.76 & 0.73 & 0.53 & 0.77 & 0.71 & 0.50 \\
\hline Fraction unbound in human plasma & PUBCHEM & 0.60 & 0.46 & 0.35 & 0.63 & 0.44 & 0.34 \\
\hline Fraction unbound in the brain & PUBCHEM & 0.48 & 0.58 & 0.46 & 0.56 & 0.56 & 0.45 \\
\hline Human liver microsomal clearance & $\mathrm{KR}$ & 0.51 & 1.08 & 0.80 & 0.56 & 1.05 & 0.79 \\
\hline Mouse liver microsomal clearance & AT2D & 0.52 & 1.21 & 0.92 & 0.53 & 1.16 & 0.88 \\
\hline Rat liver microsomal clearance & $\mathrm{KR}$ & 0.64 & 1.08 & 0.83 & 0.67 & 1.01 & 0.76 \\
\hline CACO-2 permeability & FCFP4 & 0.44 & 0.68 & 0.46 & 0.42 & 0.69 & 0.46 \\
\hline$p K_{a}$ & ECFP2 & 0.71 & 1.85 & 1.15 & 0.74 & 1.78 & 1.11 \\
\hline MDCK cell line permeability & ECFP4 & 0.62 & 0.61 & 0.44 & 0.68 & 0.56 & 0.39 \\
\hline Human renal clearance & MACCS & 0.25 & 0.54 & 0.43 & 0.27 & 0.53 & 0.42 \\
\hline Hemolytic toxicity $\left(\log H D_{50}\right)$ & ASP & 0.68 & 0.47 & 0.35 & 0.68 & 0.44 & 0.34 \\
\hline
\end{tabular}

The values reported are the squared correlation $\left(R^{2}\right)$, RMSE and MAE (average of 3 independent runs) for the calibration/validation sets

For regression models, the performance was assessed using the squared regression coefficient $\left(R^{2}\right)$ for the correlation between experimental and predicted values. the root mean squared error (RMSE) and the mean absolute error (MAE). For classification models, metrics that are sensitive to the class imbalance have been used. These include the balanced accuracy (BACC) given by:

$$
B A C C=\frac{1}{m} \sum_{i}^{m} \frac{k_{i}}{n_{i}}
$$

where $k_{i}$ is the number of correct predictions in class $i, m$ is the number of classes and $n_{i}$ is the number of examples in class $i$. In addition, other metrics such as the overall accuracy, the sensitivity (the true positive rate-TPR) and specificity (the true negative rate-TNR) and the area under the curve (AUC) are also reported (see Additional file 1).

Every model has a finite applicability domain (AD) within which its predictions can be trusted. For regression models, we quantify the prediction intervals (95\%) using the quantile regression forests approach [27]. Here, a shorter prediction interval indicates the higher stability of prediction. In the case of classification, two values: confidence and credibility are associated with the predicted label based on the conformal prediction framework [28, 29]. While the confidence provides a measure of how likely a prediction is compared to all other possible classifications, the credibility measure (equal to the highest $p$-value of any one of the possible classifications being the true label) provides an indication of how good the training set is for classifying the given example.

\section{Results and discussion}

For the various endpoints, the relevant performance metrics associated with the best fingerprint-based models are summarized in Tables 4 (for classification models) and 5 (for regression models). The complete performance summary for the training and validations sets is listed in Additional file 1: Tables S1 and S2. For all cases, permutation tests confirmed ( $\mathrm{p}$-values $<0.001)$ that the probability that the model was obtained by chance is quite low. Overall, high classification accuracies $(B A C C>0.80)$ are obtained for the blood brain barrier permeability, plasma protein binding, CYP450 inhibition (3A4/2C19/1A2/2C9/2C8 isoforms), human intestinal absorption, breast cancer resistance protein inhibition, p-glycoprotein inhibitor/ substrate and hemolytic/respiratory toxicity. For some of the other endpoints such as the mitochondrial/urinary tract toxicity, human liver microsomal stability, 


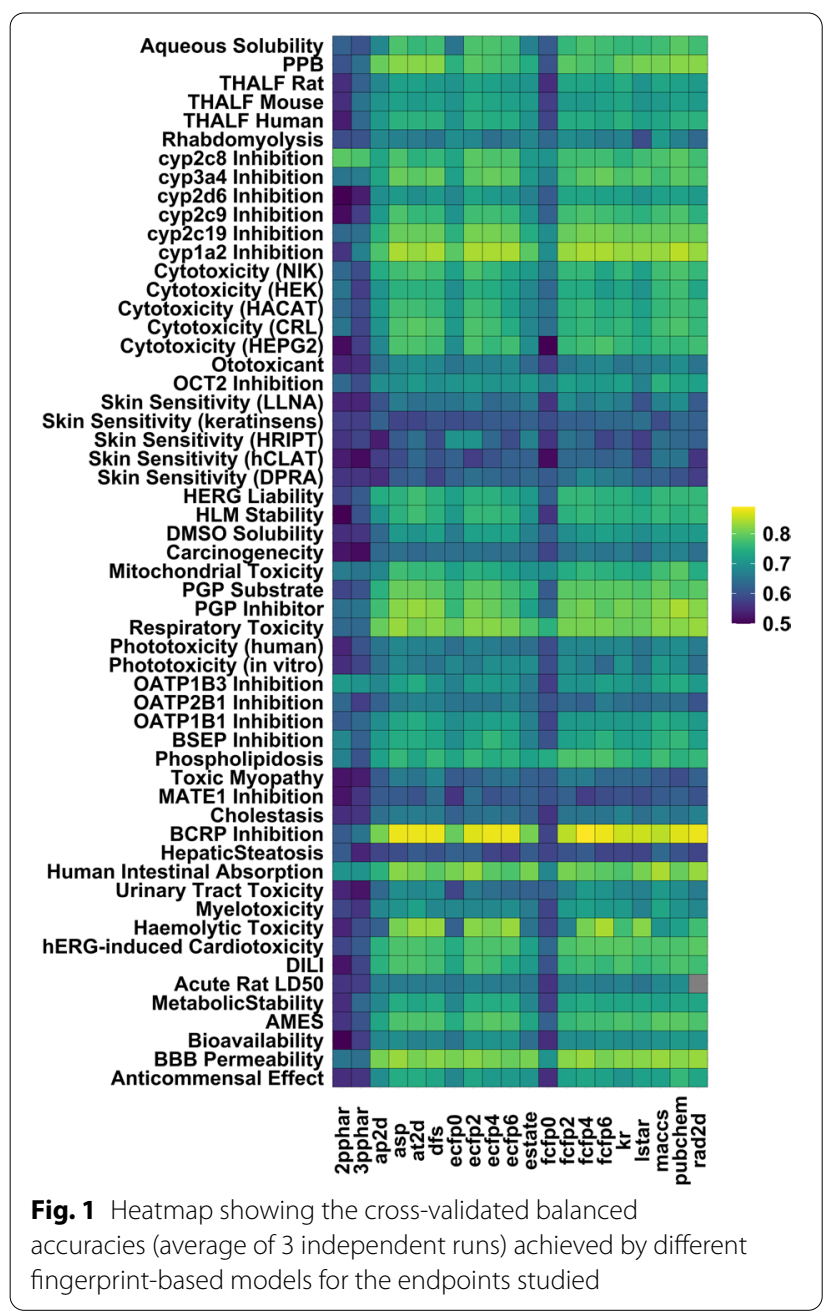

metabolic intrinsic clearance, AMES mutagenecity, cytotoxicity (multiple cell lines), hERG cardiotoxicity/ liability, drug induced liver injury, myelotoxicity, phospholipidosis, rhabdomyolysis, OATP1B1/OATP1B3 inhibition, BSEP and OCT2 inhibition, moderate ( $B A C C=0.71$ to -0.78 ) performances were observed. Properties such as skin sensitization, acute oral toxicity, phototoxicity in humans, ototoxicity, choleostasis, hepatic steatosis, and carcinogenecity yielded somewhat average results. In the case of regression models, performances were largely on the poorer side with the exception of $\mathrm{pK}_{a}, \log \mathrm{S}, \log \mathrm{D}$, human serum albumin and skin penetration, $R_{c v}^{2}>0.70$.

To identify which of the fingerprints perform well on the different datasets, we plotted heatmaps (see Figs. 1 and 2) of the balanced accuracies (for classification models) and squared correlations (in the case of regression) obtained for the different endpoints. While the pharmacaphore fingerprints (2PPHAR/3PPHAR) perform poorly on all datasets, fingerprints based on substructure keys (PUBCHEM, MACCS, KR) show moderate to high accuracies for a majority of the modelled endpoints. Although the performances for regression models are somewhat less encouraging, here too the $R_{c v}^{2}$ for PUBCHEM, ECFP4, and ASP fingerprints yield better models than the other fingerprints tested.

We further compared the performances achieved by the fingerprint models with those obtained for the $2 \mathrm{D} / 3 \mathrm{D}$ descriptor based approaches. The barplots in Fig. 3 compare the accuracies achieved by the fingerprint models with values reported by the models published earlier. While results for most properties are comparable, for some endpoints such as myelotoxicity, ototoxicity, myopathy accuracies obtained using 2D/3D descriptors are only marginally better. Indeed better results are obtained for rhabdomyolysis, phospholipidosis, phototoxicity with other descriptor based models. For phototoxicity in particular, quantum chemistry-based 3D descriptors are used which can add to the time taken. It must however be pointed out that some of the better performing models take advantage of deep learning. Attempts to improve results for selected properties were carried out using support vector machines. However, the models were not always found to improve on the random forest approach.

For the regression models calculated for selected properties: $\mathrm{pK}_{a}, \log \mathrm{S}, \log \mathrm{D}$, skin penetration, human serum albumin, MDCK permeability $\mathrm{HD}_{50}$, we assessed the prediction reliability based on the prediction intervals. Plots of the prediction intervals with respect to the observed response values for the test sets (see Additional file 1: Figure S1) showed that most of the samples lie within the $95 \%$ prediction interval which indicates that the constructed prediction intervals are reliable. For classification models, we focused on excluding compounds whose labels are predicted with low confidence and credibility. Thus, different thresholds for $p$-values (0.5, $0.6,0.7,0.8,0.9)$ were applied and the corresponding fraction of molecules that would be withheld from further testing was recorded. A plot of the overall error rates and the percentage reduction in compounds excluded from further processing (see Additional file 1: Figure S2) shows that for many of the endpoints modelled, the predictive performance is not significantly impacted even at cutoffs of 0.50 . Such a strategy that allows for compound selection based on static thresholds for the confidence/credibility offer a way to reduce the number of compounds that typically undergo experimental testing. 


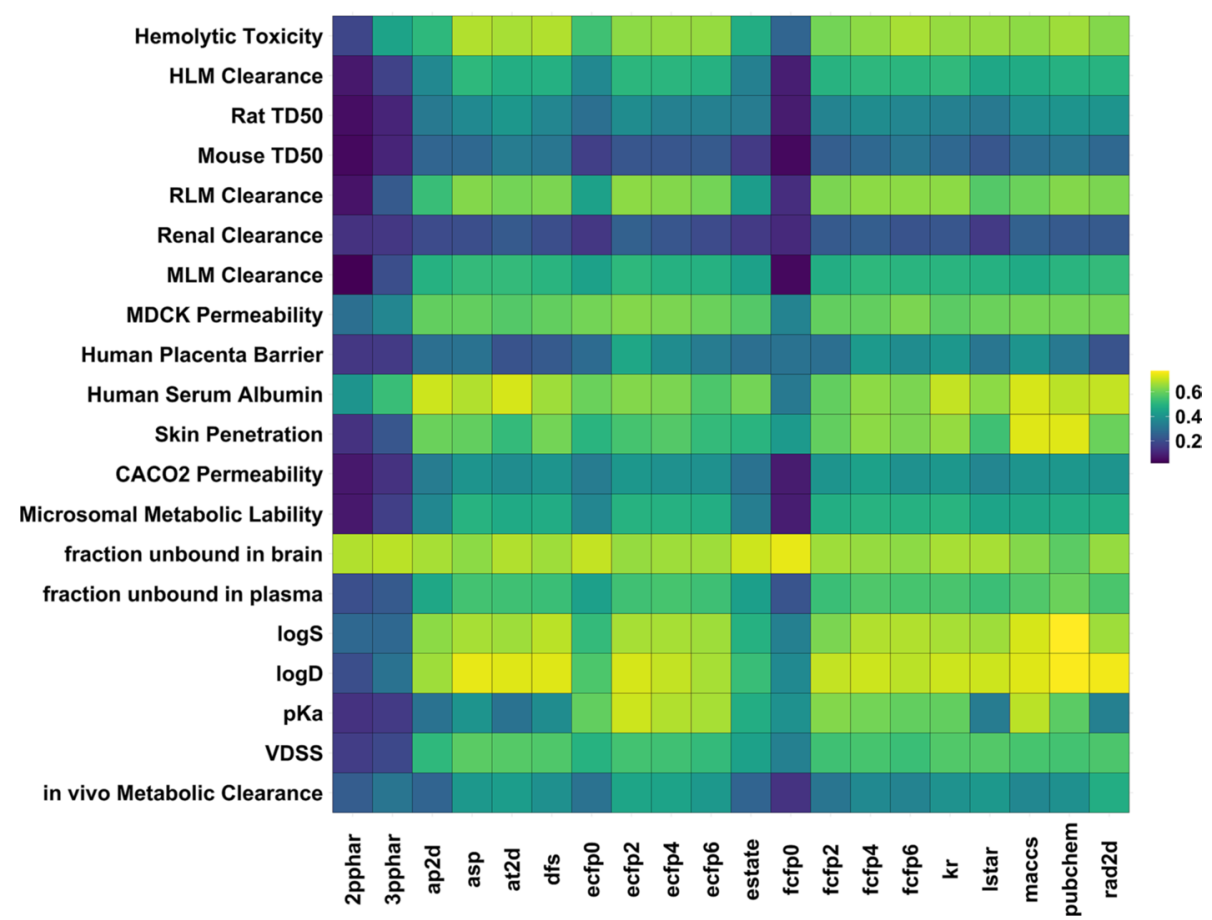

Fig. 2 Heatmap showing the cross-validated correlation coefficients (average of 3 independent runs) achieved by different fingerprint-based models for the endpoints studied

\section{Software usage}

FP-ADMET is available as open access software (GNU GPL v3.0) and can be downloaded from https://gitlab. $\mathrm{com} / \mathrm{vishsoft/fpadmet}$. Use of FP-ADMET proceeds in two steps (i) fingerprint calculation followed by (ii) predicting the ADMET endpoint of interest. The software is command line driven and is governed by a shell script (runadmet.sh) that can be run as:

bash runadmet.sh -f molecule.smi -p \#\#-a

The input to the script is a file (molecule.smi) containing SMILES strings. The \#\# is a number between 1 (predict Anticommensal Effect) and 56 (predict skin penetration) and corresponds to the prediction task. The results are written to a text file where each line contains molecule name and the predicted response. The "-a" option allows for the calculation of prediction intervals (in the case of regression) and confidence (for classification). For classification, conformal prediction is used to calculate a confidence (how certain the model is that the prediction is a singleton) and a credibility. For example, predicting AMES mutagenecity (task number 4 ) for a series of molecules produces the following results (see Table 6). The label "inactive" for compound G00001 suggests that the compound is predicted to be non-mutagenic. A confidence value of 0.95 suggests that the classifier is quite certain that the prediction is likely to be a single label. A relatively low value of credibility (0.57) suggests that the compounds like G00001 are not sufficiently represented in the training set and that the user needs to treat the prediction with caution. In the case of regression, a 95\% prediction interval (predictions at the 0.025 and 97.5 percentiles for $p K_{a}$ ) is calculated and provides a range for the predictions on an individual observation.

Table 6 Example showing the property ( $p K_{a}$ and anticommensal effect) predictions and associated uncertainties for 3 molecules

\begin{tabular}{llllrrr}
\hline Name & Anticommensal effect & Confidence & Credibility & $\boldsymbol{p} \hat{\boldsymbol{K}}_{\boldsymbol{a}}$ & $\mathbf{Q = 0 . 0 2 5}$ & $\mathbf{Q}=\mathbf{0 . 9 7 5}$ \\
\hline G00001 & Inactive & 0.95 & 0.57 & 9.62 & 4.89 & 11.49 \\
G00002 & Active & 0.95 & 0.51 & 4.41 & -1.60 & 13.06 \\
G00003 & Inactive & 0.95 & 0.57 & 3.37 & 1.66 & 6.10
\end{tabular}

$Q=0.025$ and $Q=0.975$ are the predictions calculated at percentiles 0.025 and 0.975 and allow for $95 \%$ prediction intervals 


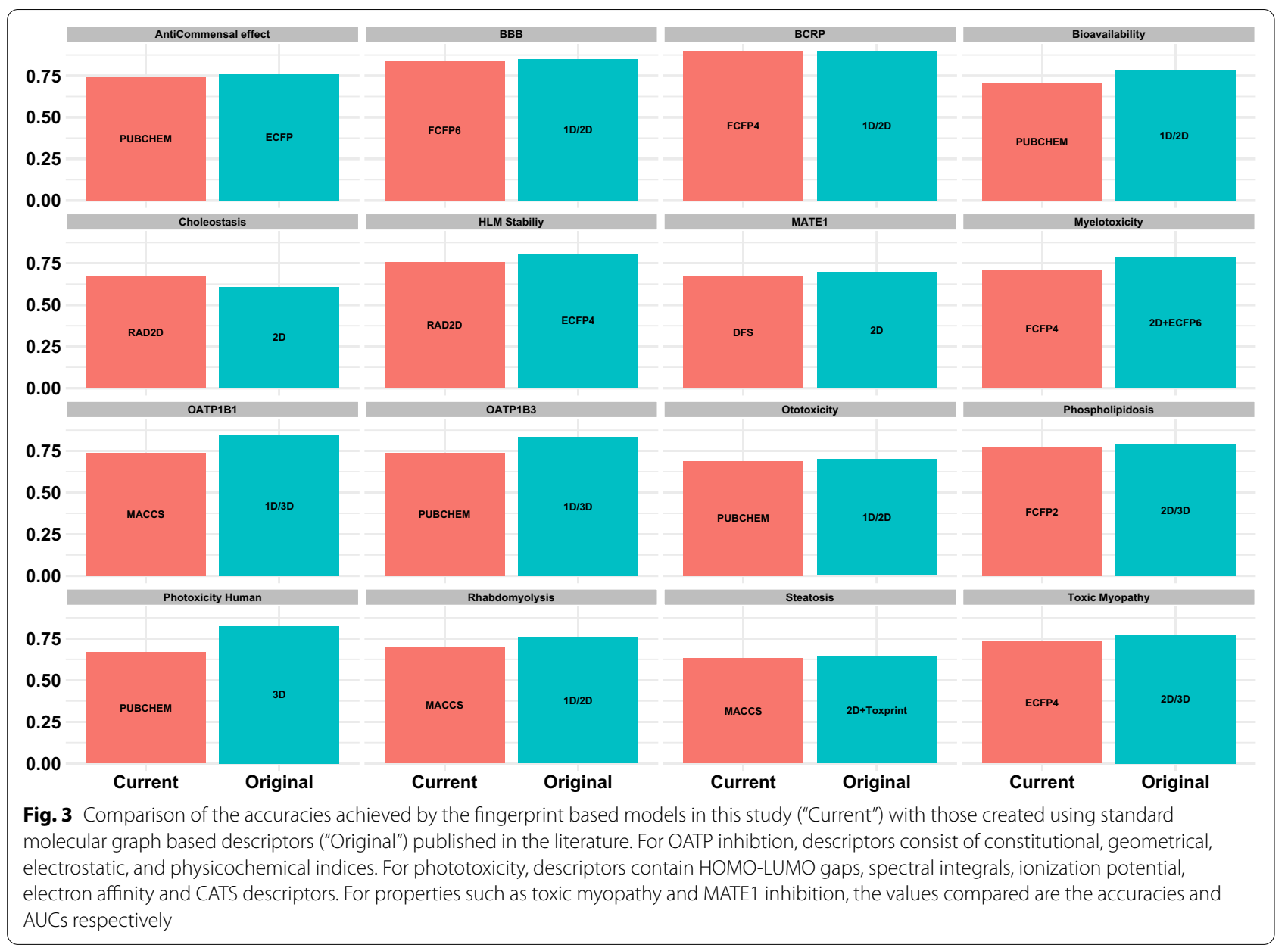

Narrow prediction intervals indicate a lower uncertainty associated with the prediction.

\section{Conclusion}

In this article, we have evaluated the performance of various molecular fingerprints for predicting a number of ADMET and ADMET-related endpoints. A total of 1500 models were analysed spanning 75 responses and 20 fingerprints. The results show that the machine learning performance using the different fingerprint encodings rival those of traditional descriptor-based methods. Future work will focus on combining different data sets in a multitask modeling approach which has been shown to yield statistically superior results compared with single-task models $[12,30]$. In order to facilitate ADMET evaluation, the best performing models have been compiled into an open access software package called FPADMET that can be downloaded from https://gitlab.com/vishsoft/ fpadmet.

\section{Supplementary Information}

The online version contains supplementary material available at https://doi. org/10.1186/s13321-021-00557-5.

Additional file 1. File contains brief descriptions of the properties modelled, additional performance statistics and figures referred to in the text.

\section{Acknowledgements}

The author thanks Dr. Amitava Roy (NIH) and Assoc. Prof. Travis Wheeler (University of Montana) for fruitful discussions.

\section{Authors' contributions}

W conceived and designed the study, performed the data analysis and wrote the paper. The author read and approved the final manuscript.

\section{Funding}

This work was supported through a grant (Grant No. 262152) from the Research Council of Norway.

\section{Declarations}

\section{Competing interests}

The authors declare that they have no competing interests. 
Received: 7 April 2021 Accepted: 20 September 2021

Published online: 28 September 2021

\section{References}

1. Ferreira LLG, Andricopulo AD (2019) ADMET modeling approaches in drug discovery. Drug Discov Today 24(5):1157-1165. https://doi.org/10. 1016/j.drudis.2019.03.015

2. Kar S, Leszczynski I (2020) Open access in silico tools to predict the ADMET profiling of drug candidates. Expert Opin Drug Discov 15(12):1473-1487. https://doi.org/10.1080/17460441.2020.1798926

3. Sushko I, Novotarskyi S, Körner R, Pandey AK, Rupp M, Teetz W, Brandmaier S, Abdelaziz A, Prokopenko V, Tanchuk VY, Todeschini R, Varnek A, Marcou G, Ertl P, Potemkin V, Grishina M, Gasteiger J, Schwab C, Baskin II, Palyulin VA, Radchenko EV, Welsh WJ, Kholodovych V, Chekmarev D, Cherkasov A, Aires-de-Sousa J, Zhang Q-Y, Bender A, Nigsch F, Patiny L, Williams A, Tkachenko V, Tetko IV (2011) Online chemical modeling environment (OCHEM): web platform for data storage, model development and publishing of chemical information. J Comput Aided Mol Des 25(6):533-554. https://doi.org/10.1007/s10822-011-9440-2

4. Guan L, Yang H, Cai Y, Sun L, Di P, Li W, Liu G, Tang Y (2019) ADMETscore-a comprehensive scoring function for evaluation of chemical drug-likeness. MedChemComm 10(1):148-157. https://doi.org/10.1039/ c8md00472b

5. Jia C-Y, Li J-Y, Hao G-F, Yang G-F (2020) A drug-likeness toolbox facilitates ADMET study in drug discovery. Drug Discov Today 25(1):248-258. https://doi.org/10.1016/j.drudis.2019.10.014

6. Pires DEV, Blundell TL, Ascher DB (2015) pkCSM: predicting smallmolecule pharmacokinetic and toxicity properties using graph-based signatures. J Med Chem 58(9):4066-4072. https://doi.org/10.1021/acs. jmedchem.5b00104

7. Clark AM, Dole K, Coulon-Spektor A, McNutt A, Grass G, Freundlich JS, Reynolds RC, Ekins S (2015) Open source Bayesian models. 1. Application to ADME/Tox and drug discovery datasets. J Chem Inf Model 55(6):12311245. https://doi.org/10.1021/acs.jcim.5b00143

8. Lagorce D, Bouslama L, Becot J, Miteva MA, Villoutreix BO (2017) FAFdrugs4: free ADME-Tox filtering computations for chemical biology and early stages drug discovery. Bioinformatics 33(22):3658-3660. https://doi. org/10.1093/bioinformatics/btx491

9. Daina A, Michielin O, Zoete V (2017) SwissADME: a free web tool to evaluate pharmacokinetics, drug-likeness and medicinal chemistry friendliness of small molecules. Sci Rep. https://doi.org/10.1038/srep42717

10. Schyman P, Liu R, Desai V, Wallqvist A (2017) vNN web server for ADMET predictions. Front Pharmacol. https://doi.org/10.3389/fphar.2017.00889

11. Yang H, Lou C, Sun L, Li J, Cai Y, Wang Z, Li W, Liu G, Tang Y (2018) admetSAR 2.0: web-service for prediction and optimization of chemical ADMET properties. Bioinformatics 35(6):1067-1069. https://doi.org/10.1093/bioin formatics/bty707

12. Xiong G, Wu Z, Yi J, Fu L, Yang Z, Hsieh C, Yin M, Zeng X, Wu C, Lu A, Chen X, Hou T, Cao D (2021) ADMETlab 2.0: an integrated online platform for accurate and comprehensive predictions of ADMET properties. Nucleic Acids Res 49(W1):5-14. https://doi.org/10.1093/nar/gkab255

13. Banerjee P, Eckert AO, Schrey AK, Preissner R (2018) ProTox-II: a webserver for the prediction of toxicity of chemicals. Nucleic Acids Res 46(W1):257263. https://doi.org/10.1093/nar/gky318

14. Patel RD, Kumar SP, Pandya HA, Solanki HA (2018) MDCKpred: a webtool to calculate MDCK permeability coefficient of small molecule using membrane-interaction chemical features. Toxicol Mech Methods 28(9):685-698. https://doi.org/10.1080/15376516.2018.1499840

15. Zhang L, Ai H, Chen W, Yin Z, Hu H, Zhu J, Zhao J, Zhao Q, Liu H (2017) CarcinoPred-EL: novel models for predicting the carcinogenicity of chemicals using molecular fingerprints and ensemble learning methods. Sci Rep. https://doi.org/10.1038/s41598-017-02365-0

16. Wang Y-W, Huang L, Jiang S-W, Li K, Zou J, Yang S-Y (2020) CapsCarcino: a novel sparse data deep learning tool for predicting carcinogens. Food Chem Toxicol 135:110921. https://doi.org/10.1016/j.fct.2019.110921

17. Yap CW (2010) PaDEL-descriptor: an open source software to calculate molecular descriptors and fingerprints. J Comp Chem 32(7):1466-1474. https://doi.org/10.1002/jcc.21707
18. Venkatraman V, Alsberg BK (2016) KRAKENX: software for the generation of alignment-independent 3D descriptors. J Mol Model. https://doi.org/ 10.1007/s00894-016-2957-5

19. Muegge I, Mukherjee P (2015) An overview of molecular fingerprint similarity search in virtual screening. Expert Opin Drug Discov 11(2):137-148. https://doi.org/10.1517/17460441.2016.1117070

20. Cereto-Massagué A, Ojeda MJ, Valls C, Mulero M, Garcia-Vallvé S, Pujadas $G$ (2015) Molecular fingerprint similarity search in virtual screening. Methods 71:58-63. https://doi.org/10.1016/j.ymeth.2014.08.005

21. Hinselmann G, Rosenbaum L, Jahn A, Fechner N, Zell A (2011) jCompoundMapper: an open source java library and command-line tool for chemical fingerprints. J Cheminf. https://doi.org/10.1186/1758-2946-3-3

22. Willighagen EL, Mayfield JW, Alvarsson J, Berg A, Carlsson L, Jeliazkova N, Kuhn S, Pluskal T, Rojas-Chertó M, Spjuth O, Torrance G, Evelo CT, Guha R, Steinbeck C (2017) The chemistry development kit (CDK) v2.0: atom typing, depiction, molecular formulas, and substructure searching. J Cheminf. https://doi.org/10.1186/s13321-017-0220-4

23. Breiman L (2001) Random forests. Mach Learn 45(1):5-32. https://doi.org/ 10.1023/a:1010933404324

24. Wright MN, Ziegler A (2017) ranger: a fast implementation of random forests for high dimensional data in C++ and R. J Stat Soft 77(1):1-17. https://doi.org/10.18637/jss.v077.i01

25. R Core Team (2020) R: a language and environment for statistical computing. R Foundation for Statistical Computing, Vienna, Austria. R Foundation for Statistical Computing. https://www.R-project.org/

26. Chawla NV, Bowyer KW, Hall LO, Kegelmeyer WP (2002) SMOTE synthetic minority over-sampling technique. J Artif Intell Res 16:321-357. https:// doi.org/10.1613/jair.953

27. Meinshausen N (2006) Quantile regression forests. J Mach Learn Res 7(35):983-999

28. Papadopoulos H (2008) Chap. 18. Inductive conformal prediction: theory and application to neural networks. In: Fritzsche P (ed) Tools in artificial intelligence. IntechOpen, Rijeka. https://doi.org/10.5772/6078

29. Ahlberg E, Hammar O, Bendtsen C, Carlsson L (2017) Current application of conformal prediction in drug discovery. Ann Math Artif Intell 81(1-2):145-154. https://doi.org/10.1007/s10472-017-9550-1

30. Wenzel J, Matter H, Schmidt F (2019) Predictive multitask deep neural network models for ADME-Tox properties: learning from large data sets. J Chem Inf Model 59(3):1253-1268. https://doi.org/10.1021/acs.jcim.8b007 85

31. Shaker B, Yu M-S, Song JS, Ahn S, Ryu JY, Oh K-S, Na D (2020) LightBBB: computational prediction model of blood-brain-barrier penetration based on LightGBM. Bioinformatics. https://doi.org/10.1093/bioinforma tics/btaa918

32. Falcón-Cano G, Molina C, Cabrera-Pérez MÁ (2020) ADME prediction with KNIME: development and validation of a publicly available workflow for the prediction of human oral bioavailability. J Chem Inf Model 60(6):2660-2667. https://doi.org/10.1021/acs.jcim.0c00019

33. Maier L, Pruteanu M, Kuhn M, Zeller G, Telzerow A, Anderson EE, Brochado AR, Fernandez KC, Dose H, Mori H, Patil KR, Bork P, Typas A (2018) Extensive impact of non-antibiotic drugs on human gut bacteria. Nature 555(7698):623-628. https://doi.org/10.1038/nature25979

34. Zheng S, Chang W, Liu W, Liang G, Xu Y, Lin F (2018) Computational prediction of a new ADMET endpoint for small molecules: anticommensal effect on human gut microbiota. J Chem Inf Model 59(3):1215-1220. https://doi.org/10.1021/acs.jcim.8b00600

35. Veith $H$, Southall $N$, Huang $R$, James $T$, Fayne D, Artemenko N, Shen M, Inglese J, Austin CP, Lloyd DG, Auld DS (2009) Comprehensive characterization of cytochrome p450 isozyme selectivity across chemical libraries. Nat Biotechnol 27(11):1050-1055. https://doi.org/10.1038/nbt.1581

36. Zhang X, Zhao P, Wang Z, Xu X, Liu G, Tang Y, Li W (2021) In silico prediction of CYP2C8 inhibition with machine-learning methods. Chem Res Toxicol 34(8):1850-1859. https://doi.org/10.1021/acs.chemrestox.1 1 00078

37. Wang N-N, Huang C, Dong J, Yao Z-J, Zhu M-F, Deng Z-K, Lv B, Lu A-P, Chen AF, Cao D-S (2017) Predicting human intestinal absorption with modified random forest approach: a comprehensive evaluation of molecular representation, unbalanced data, and applicability domain issues. RSC Adv 7(31):19007-19018. https://doi.org/10.1039/c6ra28442f

38. Jiang D, Lei T, Wang Z, Shen C, Cao D, Hou T (2020) ADMET evaluation in drug discovery. 20. Prediction of breast cancer resistance protein 
inhibition through machine learning. J Cheminf. https://doi.org/10. 1186/s13321-020-00421-y

39. Esaki T, Watanabe R, Kawashima H, Ohashi R, Natsume-Kitatani Y, Nagao C, Mizuguchi K (2018) Data curation can improve the prediction accuracy of metabolic intrinsic clearance. Mol Inf 38(1-2):1800086. https://doi.org/10.1002/minf.201800086

40. Liu R, Schyman P, Wallqvist A (2015) Critically assessing the predictive power of QSAR models for human liver microsomal stability. J Chem Inf Model 55(8):1566-1575. https://doi.org/10.1021/acs.jcim.5b00255

41. Wang P-H, Tu Y-S, Tseng YJ (2019) PgpRules: a decision tree based prediction server for p-glycoprotein substrates and inhibitors. Bioinformatics 35(20):4193-4195. https://doi.org/10.1093/bioinformatics/ btz213

42. Tetko IV, Novotarskyi S, Sushko I, Ivanov V, Petrenko AE, Dieden R, Lebon F, Mathieu B (2013) Development of dimethyl sulfoxide solubility models using 163000 molecules: using a domain applicability metric to select more reliable predictions. J Chem Inf Model 53(8):1990-2000. https://doi.org/10.1021/ci400213d

43. Perryman AL, Inoyama D, Patel JS, Ekins S, Freundlich JS (2020) Pruned machine learning models to predict aqueous solubility. ACS Omega 5(27):16562-16567. https://doi.org/10.1021/acsomega.0c01251

44. Borba JVB, Braga RC, Alves VM, Muratov EN, Kleinstreuer N, Tropsha A, Andrade CH (2020) Pred-skin: a web portal for accurate prediction of human skin sensitizers. Chem Res Toxicol. https://doi.org/10.1021/acs. chemrestox.0c00186

45. Gadaleta D, Vuković K, Toma C, Lavado GJ, Karmaus AL, Mansouri K, Kleinstreuer NC, Benfenati E, Roncaglioni A (2019) SAR and QSAR modeling of a large collection of LD50 rat acute oral toxicity data. J Cheminf. https:// doi.org/10.1186/s13321-019-0383-2

46. Xu C, Cheng F, Chen L, Du Z, Li W, Liu G, Lee PW, Tang Y (2012) In silico prediction of chemical Ames mutagenicity. J Chem Inf Model 52(11):2840-2847. https://doi.org/10.1021/ci300400a

47. Sun H, Wang Y, Cheff DM, Hall MD, Shen M (2020) Predictive models for estimating cytotoxicity on the basis of chemical structures. Bioorg Med Chem 28(10):115422. https://doi.org/10.1016/j.bmc.2020.115422

48. Mora JR, Marrero-Ponce Y, García-Jacas CR, Causado AS (2020) Ensemble models based on QuBiLS-MAS features and shallow learning for the prediction of drug-induced liver toxicity: improving deep learning and traditional approaches. Chem Res Toxicol 33(7):1855-1873. https://doi. org/10.1021/acs.chemrestox.0c00030

49. Zheng S, Wang Y, Liu W, Chang W, Liang G, Xu Y, Lin F (2019) In silico prediction of hemolytic toxicity on the human erythrocytes for small molecules by machine-learning and genetic algorithm. J Med Chem 63(12):6499-6512. https://doi.org/10.1021/acs.jmedchem.9b00853

50. Cai C, Guo P, Zhou Y, Zhou J, Wang Q, Zhang F, Fang J, Cheng F (2019) Deep learning-based prediction of drug-induced cardiotoxicity. J Chem Inf Model 59(3):1073-1084. https://doi.org/10.1021/acs.jcim.8b00769

51. Siramshetty VB, Nguyen D-T, Martinez NJ, Southall NT, Simeonov A, Zakharov AV (2020) Critical assessment of artificial intelligence methods for prediction of hERG channel inhibition in the "big data" era. J Chem Inf Model 60(12):6007-6019. https://doi.org/10.1021/acs.jcim.0c00884

52. Hemmerich J, Troger F, Füzi B, Ecker FG (2020) Using machine learning methods and structural alerts for prediction of mitochondrial toxicity. Mol Inf 39(5):2000005. https://doi.org/10.1002/minf.202000005

53. Lei T, Sun H, Kang Y, Zhu F, Liu H, Zhou W, Wang Z, Li D, Li Y, Hou T (2017) ADMET evaluation in drug discovery. 18. Reliable prediction of chemicalinduced urinary tract toxicity by boosting machine learning approaches. Mol Pharm 14(11):3935-3953. https://doi.org/10.1021/acs.molpharmac eut.7b00631

54. Zhang H, Ren J-X, Ma J-X, Ding L (2018) Development of an in silico prediction model for chemical-induced urinary tract toxicity by using Naïve Bayes classifier. Mol Divers 23(2):381-392. https://doi.org/10.1007/ s1 1030-018-9882-8

55. Schmidt F, Wenzel J, Halland N, Güssregen S, Delafoy L, Czich A (2019) Computational investigation of drug phototoxicity: photosafety assessment, photo-toxophore identification, and machine learning. Chem Res Toxicol 32(11):2338-2352. https://doi.org/10.1021/acs.chemrestox.9b003 38

56. Hu X, Yan A (2011) In silico models to discriminate compounds inducing and noninducing toxic myopathy. Mol Inf 31(1):27-39. https://doi.org/10. 1002/minf.201100067
57. Zhang H, Yu P, Zhang T-G, Kang Y-L, Zhao X, Li Y-Y, He J-H, Zhang J (2015) In silico prediction of drug-induced myelotoxicity by using Naiive Bayes method. Mol Divers 19(4):945-953. https://doi.org/10.1007/ s1 1030-015-9613-3

58. Fusani L, Brown M, Chen H, Ahlberg E, Noeske T (2017) Predicting the risk of phospholipidosis with in silico models and an image-based in vitro screen. Mol Pharm 14(12):4346-4352. https://doi.org/10.1021/acs.molph armaceut.7b00388

59. Kotsampasakou E, Ecker GF (2017) Predicting drug-induced cholestasis with the help of hepatic transporters - an in silico modeling approach. J Chem Inf Model 57(3):608-615. https://doi.org/10.1021/acs.jcim.6b00518

60. Cui X, Liu J, Zhang J, Wu Q, Li X (2019) In silico prediction of drug-induced rhabdomyolysis with machine-learning models and structural alerts. J Appl Toxicol 39(8):1224-1232. https://doi.org/10.1002/jat.3808

61. Zhang H, Ma J-X, Liu C-T, Ren J-X, Ding L (2018) Development and evaluation of in silico prediction model for drug-induced respiratory toxicity by using Naïve Bayes classifier method. Food Chem Toxicol 121:593-603. https://doi.org/10.1016/j.fct.2018.09.051

62. Zhang H, Liu C-T, Mao J, Shen C, Xie R-L, Mu B (2020) Development of novel in silico prediction model for drug-induced ototoxicity by using Naiive Bayes classifier approach. Toxicol In Vitro 65:104812. https://doi.org/ 10.1016/j.tiv.2020.104812

63. Wittwer MB, Zur AA, Khuri N, Kido Y, Kosaka A, Zhang X, Morrissey KM, Sali A, Huang Y, Giacomini KM (2013) Discovery of potent, selective multidrug and toxin extrusion transporter 1 (MATE1, SLC47a1) inhibitors through prescription drug profiling and computational modeling. J Med Chem 56(3):781-795. https://doi.org/10.1021/jm301302s

64. Jain S, Norinder U, Escher SE, Zdrazil B (2020) Combining in vivo data with in silico predictions for modeling hepatic steatosis by using stratified bagging and conformal prediction. Chem Res Toxicol. https://doi.org/10. 1021/acs.chemrestox.0c00511

65. Türková A, Jain S, Zdrazil B (2018) Integrative data mining, scaffold analysis, and sequential binary classification models for exploring ligand profiles of hepatic organic anion transporting polypeptides. J Chem Inf Model 59(5):1811-1825. https://doi.org/10.1021/acs.jcim.8b00466

66. McLoughlin KS, Jeong CG, Sweitzer TD, Minnich AJ, Tse MJ, Bennion BJ, Allen JE, Calad-Thomson S, Rush TS, Brase JM (2021) Machine learning models to predict inhibition of the bile salt export pump. J Chem Inf Model 61 (2):587-602. https://doi.org/10.1021/acs.jcim.0c00950

67. Kido Y, Matsson P, Giacomini KM (2011) Profiling of a prescription drug library for potential renal drug-drug interactions mediated by the organic cation transporter 2. J Med Chem 54(13):4548-4558. https://doi. org/10.1021/jm2001629

68. Yuan Y, Chang S, Zhang Z, Li Z, Li S, Xie P, Yau W-P, Lin H, Cai W, Zhang Y, Xiang X (2020) A novel strategy for prediction of human plasma protein binding using machine learning techniques. Chemom Intell Lab Syst 199:103962. https://doi.org/10.1016/j.chemolab.2020.103962

69. Podlewska S, Kafel R (2018) MetStabOn—online platform for metabolic stability predictions. Int J Mol Sci 19(4):1040. https://doi.org/10.3390/ ijms 19041040

70. Sorkun MC, Khetan A, Er S (2019) AqSolDB, a curated reference set of aqueous solubility and 2D descriptors for a diverse set of compounds. Sci Data. https://doi.org/10.1038/s41597-019-0151-1

71. Hsiao Y-W, Fagerholm U, Norinder U (2013) In silico categorization of in vivo intrinsic clearance using machine learning. Mol Pharm 10(4):13181321. https://doi.org/10.1021/mp300484r

72. Lindh M, Karlén A, Norinder U (2017) Predicting the rate of skin penetration using an aggregated conformal prediction framework. Mol Pharm 14(5):1571-1576. https://doi.org/10.1021/acs.molpharmaceut.7b00007

73. Serra A, Önlü S, Coretto P, Greco D (2019) An integrated quantitative structure and mechanism of action-activity relationship model of human serum albumin binding. J Cheminf. https://doi.org/10.1186/ s13321-019-0359-2

74. Ciura K, Ulenberg S, Kapica H, Kawczak P, Belka M, BączekT (2020) Drug affinity to human serum albumin prediction by retention of cetyltrimethylammonium bromide pseudostationary phase in micellar electrokinetic chromatography and chemically advanced template search descriptors. J Pharm Biomed 188:113423. https://doi.org/10.1016/j.jpba.2020.113423

75. Giaginis C, Zira A, Theocharis S, Tsantili-Kakoulidou A (2009) Application of quantitative structure activity relationships for modeling drug and chemical transport across the human placenta barrier: a multivariate data 
analysis approach. J Appl Toxicol 29(8):724-733. https://doi.org/10.1002/ jat.1466

76. Bercu JP, Morton SM, Deahl JT, Gombar VK, Callis CM, van Lier RBL (2010) In silico approaches to predicting cancer potency for risk assessment of genotoxic impurities in drug substances. Regul Toxicol Pharmacol 57(2):300-306. https://doi.org/10.1016/j.yrtph.2010.03.010

77. Simeon S, Montanari D, Gleeson MP (2019) Investigation of factors affecting the performance of in silico volume distribution QSAR models for human, rat, mouse, dog \& monkey. Mol Inf 38(10):1900059. https://doi. org/10.1002/minf.201900059

78. Fu L, Liu L, Yang Z-J, Li P, Ding J-J, Yun Y-H, Lu A-P, Hou T-J, Cao D-S (2019) Systematic modeling of $\log \mathrm{D}_{7} .4$ based on ensemble machine learning, group contribution, and matched molecular pair analysis. J Chem Inf Model 60(1):63-76. https://doi.org/10.1021/acs.jcim.9b00718

79. Watanabe R, Esaki T, Kawashima H, Natsume-Kitatani Y, Nagao C, Ohashi R, Mizuguchi K (2018) Predicting fraction unbound in human plasma from chemical structure: improved accuracy in the low value ranges. Mol Pharm 15(11):5302-5311. https://doi.org/10.1021/acs.molpharmaceut. 8 b00785

80. Esaki T, Ohashi R, Watanabe R, Natsume-Kitatani Y, Kawashima H, Nagao C, Mizuguchi K (2019) Computational model to predict the fraction of unbound drug in the brain. J Chem Inf Model 59(7):3251-3261. https:// doi.org/10.1021/acs.jcim.9b00180
81. Lu Y, Anand S, Shirley W, Gedeck P, Kelley BP, Skolnik S, Rodde S, Nguyen M, Lindvall M, Jia W (2019) Prediction of $\mathrm{pK}_{\mathrm{a}}$ using machine learning methods with rooted topological torsion fingerprints: application to aliphatic amines. J Chem Inf Model 59(11):4706-4719. https://doi.org/10. 1021/acs.jcim.9b00498

82. Mansouri K, Cariello NF, Korotcov A, Tkachenko V, Grulke CM, Sprankle CS, Allen D, Casey WM, Kleinstreuer NC, Williams AJ (2019) Open-source QSAR models for pKa prediction using multiple machine learning approaches. J Cheminf. https://doi.org/10.1186/s13321-019-0384-1

83. Chen J, Yang H, Zhu L, Wu Z, Li W, Tang Y, Liu G (2020) In silico prediction of human renal clearance of compounds using quantitative structurepharmacokinetic relationship models. Chem Res Toxicol 33(2):640-650. https://doi.org/10.1021/acs.chemrestox.9b00447

84. Zheng S, Xiong J, Wang Y, Liang G, Xu Y, Lin F (2020) Quantitative prediction of hemolytic toxicity for small molecules and their potential hemolytic fragments by mach. learn. and recursive fragmentation methods. J Chem Inf Model 60(6):3231-3245. https://doi.org/10.1021/acs.jcim.0c001 02

\section{Publisher's Note}

Springer Nature remains neutral with regard to jurisdictional claims in published maps and institutional affiliations.
Ready to submit your research? Choose BMC and benefit from:

- fast, convenient online submission

- thorough peer review by experienced researchers in your field

- rapid publication on acceptance

- support for research data, including large and complex data types

- gold Open Access which fosters wider collaboration and increased citations

- maximum visibility for your research: over $100 \mathrm{M}$ website views per year

At BMC, research is always in progress.

Learn more biomedcentral.com/submissions 